\title{
Taking a bite out of Wnts
}

\author{
Cortney M Bouldin ${ }^{1}$, David Kimelman ${ }^{1}$ \\ 'Department of Biochemistry, University of Washington, Seattle, WA 98103-7350, USA \\ Cell Research (2012) 22:1621-1623. doi:10.1038/cr.2012.104; published online 10 July 2012
}

\begin{abstract}
Proper control of intercellular communication through the Wnt signaling pathway is of critical importance for many aspects of biology, including head formation during vertebrate embryogenesis. A recent Cell paper describes the discovery of a novel protein, TIKI, which controls head size through a surprising new mechanism of Wnt antagonism.
\end{abstract}

The Wnt signaling pathway is involved in a myriad of biological processes, including embryonic development, stem cell regulation and human disease. Since the discovery of the founding family member, a framework for understanding Wnt signaling has taken shape [1]. Similar to other intercellular signaling pathways, the Wnt ligand is synthesized and secreted, and then binds to a co-receptor complex on a neighboring cell (Figure 1A). Subsequent downstream events elicit a Wnt response, which can be split into one of two main classes: a $\beta$-catenin-dependent class (canonical Wnt signaling), which involves transcriptional activation, and a $\beta$-catenin-independent class (noncanonical Wnt signaling), which is less well understood [2]. Importantly, regulation of both canonical and noncanonical Wnt signaling often occurs in the intercellular milieu through the action of antagonists. Many secreted Wnt antagonists have been shown to act

Correspondence: David Kimelman

E-mail: kimelman@uw.edu by physically interfering with various aspects of the Wnt signaling pathway. For instance, the antagonist Dickkopf (Dkk) blocks Wnt signaling by binding to the canonical pathway co-receptor of Frizzled, known as LRP [3], thus specifically blocking canonical Wnt signaling (Figure 1A). Other antagonists, such as Frzb and Cerberus, function by binding directly to the Wnt ligand to prevent Wnt signaling $[4,5]$ (Figure 1A). In a recent Cell paper, Zhang et al. [6] used a functional expression screen to identify a new method of Wnt antagonism, which acts through the newly identified TIKI to cleave Wnt proteins.

The authors' screen took advantage of a unique tissue in early stage amphibian embryos called the SpemannMangold organizer (named after its discoverers Hans Spemann and Hilde Mangold - hereafter referred to as the organizer). In a gastrulating embryo, the organizer secretes Wnt antagonists that actively work to create a Wnt-free zone, which will go on to become the head [7] (Figure 1B). By overexpressing mRNAs in early embryos from the frog (Xenopus) and characterizing changes to the resulting head, Zhang et al. identified a new modulator of Wnt signaling, which they named TIKI because its overexpression in Xenopus resulted in a large head, reminding them of the large-headed humanoids in Polynesian sculptures. TIKI is a previously unidentified transmembrane protein with no domains of obvious homology. The authors show that TIKI is expressed in the organizer of the gastrulating Xenopus embryo and that it functions when overexpressed by antagonizing Wnt signaling. Importantly, using antisense oligonucleotides to deplete TIKI from Xenopus embryos, they show that a reduction of TIKI results in embryos with diminished heads, demonstrating that TIKI is necessary for normal organizer function. These results could have been cause to include the newly identified TIKI with other known Wnt antagonists and move on, but the authors probed deeper into the mechanism of TIKI function and discovered a unique and surprising role for TIKI in antagonizing the Wnt signaling pathway.

Post-translational processing has emerged as an important aspect of Wnt signaling. Addition of a fatty acid side chain to serine 209 (S209) in human Wnt3a by the Porcupine protein is required for secretion of Wnt [8]. Intriguingly, a new structural study using Xenopus Wnt8 (Xwnt8) reveals that the addition of a palmitoleic or palmitic acid to S209 is essential for Wnt's interaction with the Frizzled receptor, forming part of a domain that produces a high-affinity interaction with the Wnt-binding region of Frizzled [9] (Figure 1A). Cysteine 77 (C77) had also been proposed as a site of fatty acid addition, supported by the fact that an alanine substitution at C77 (C77A) changes the solubility of Wnt [10]. While new data suggests that the C77A mutant affects solubility by aggregating Wnts and not by directly blocking palmitoylation (see below), the 

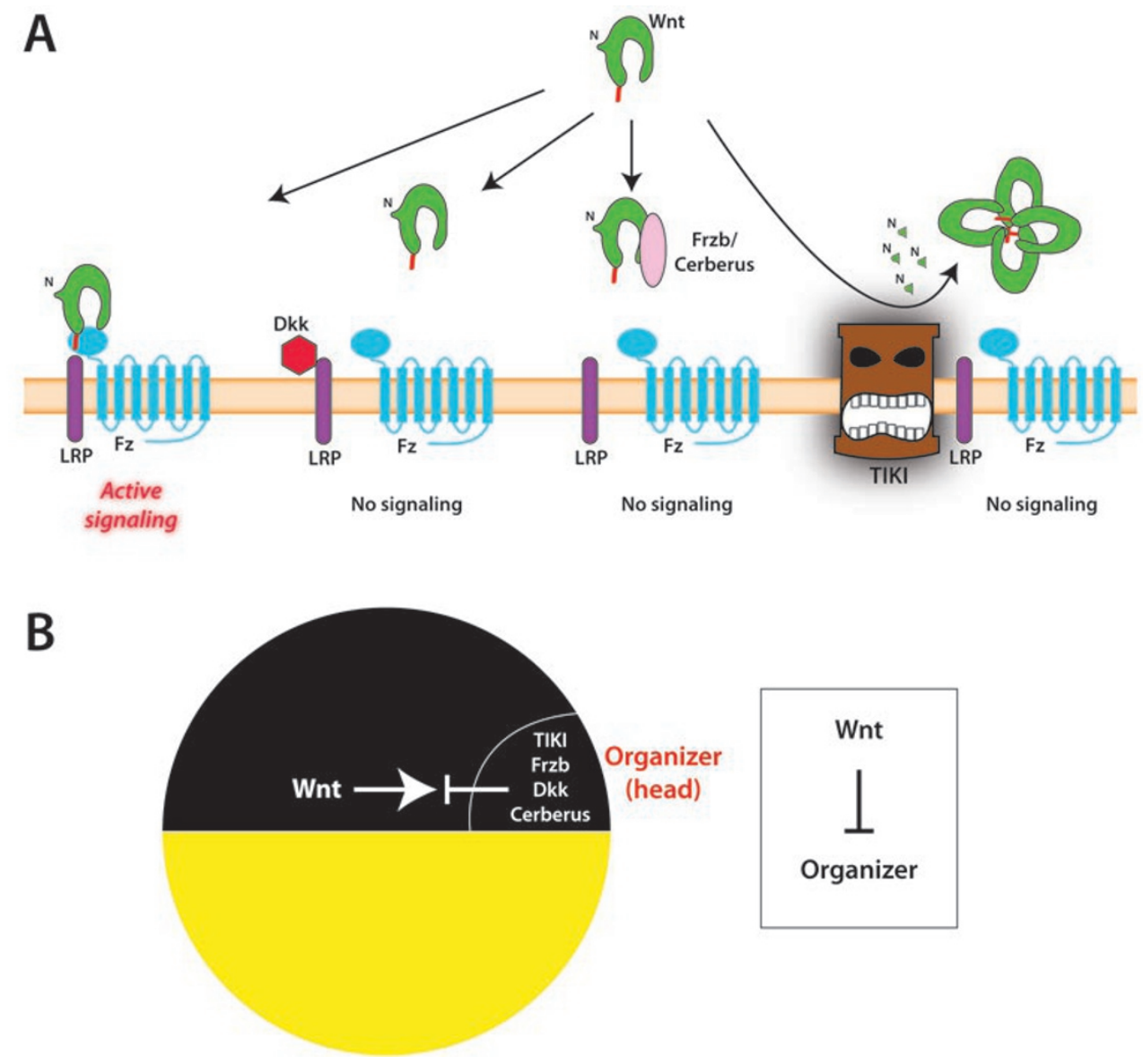

Figure 1 Antagonism of Wnt signaling is necessary for vertebrate head formation. (A) Palmitoylated Wnt binds to a complex of LRP and Fz to activate canonical Wnt signaling. Antagonists of Wnt signaling include Dkk, which binds LRP, Frzb and Cerberus, which bind Wnt, and TIKI, which proteolytically cleaves the protruding N-terminus of Wnt, causing inactive Wnt aggregates to form. (B) The Xenopus organizer, which will later become the head mesoderm, secretes four Wnt antagonists including TIKI. The role of the Wnt antagonists is to prevent the nearby produced canonical Wnt from preventing head formation, thus allowing the head to form in the region of the organizer.

C77A mutant has been previously used as a tool to study un-palmitoylated Wnt. Similar to Wnt3a with a C77A mutation, the authors show that Wnt in the presence of TIKI shifts from a detergent-soluble form to an aqueous-soluble form. Curiously, they found that the presence of TIKI did not affect palmitoylation or secretion of Wnt3a, and instead TIKI prevents Wnt3a from binding either of its co-receptors. Through a variety of methods the authors found that eight amino acids of the $\mathrm{N}$-terminus of mature human Wnt3a protein were cleaved off in a TIKI-dependent manor, whereas 16 or 20 amino acids were cleaved from the
N-terminus of XWnt8.

How could removal of a small portion of Wnt have a substantial effect on Wnt activity and solubility without affecting lipid modification? One important clue came from the observation that under non-reducing PAGE analysis, Wnt3a in the presence of TIKI creates large molecular weight oligomers of Wnt protein (Figure 1A). In contrast, PAGE under reducing conditions eliminated the Wnt oligomers, indicating that the oligomers were noncovalently linked. Since Wnt3a C77A behaved similarly, the authors proposed that removal of the $\mathrm{N}$-terminal residues created confor- mational changes in Wnt that masked the fatty acid (explaining the change in solubility) and allowed oligomerization of Wnt (explaining loss of receptor binding). However, the new structural data showing Xwnt8 bound to the Wntbinding domain of the Frizzled receptor provides an alternative explanation [9]. First, we note that the N-terminus of Wnt protrudes as a helical domain from the rest of the protein, which makes this region accessible to cleavage by TIKI. Second, the $\mathrm{C} 77$ equivalent residue in Xwnt8 is not a site of palmitolyation as previously indicated [10] and instead is engaged in a disulfide bond [9]. Thus 
the C77A mutation is likely to alter disulfide bonding since it creates an unpaired cysteine, which could then result in Wnt aggregates by interacting with cysteine residues on other Wnt molecules. Similarly, removal of the $\mathrm{N}$-terminus by TIKI might expose a buried and reduced cysteine, which could then form intermolecular disulfide bonds with other Wnts, producing Wnt oligomers.

In addition to the canonical Wnts, Wnt3a and Xwnt8, Zhang et al. demonstrate that TIKI can cleave the noncanonical Wnt, Wnt5a. Although they did not demonstrate that the cleaved Wnt5a aggregates and is non-functional, this result suggests that TIKI might regulate both arms of the Wnt pathway. Interestingly, TIKI seems to have specificity since Xwnt11 was not cleaved. The authors found that TIKI bound all Wnts that they tested, including Xwnt11, but they were unable to identify a consensus cleavage motif. It will be intriguing to see whether the difference between cleaved and non-cleaved Wnts depends on the presence or absence of a protruding N-terminus, which could explain the absence of a consensus sequence.

TIKI homologs were also identified in zebrafish (Danio), nematode (C. elegans), sea anemone (Nematostella) and sponge (Amphimedon). Curiously, the authors describe possible homologs in bacteria although the role of TIKI proteins in bacteria is unclear because other components of the Wnt signaling pathway have only been identified in multicellular organisms. While the functions of the ancestral TIKI homologs remain untested, their presence in such a diversity of genomes including the most basal organisms with Wnt signaling [11] suggests a broad reaching function for TIKI.
An important unanswered question in developmental biology is why so many antagonists are necessary within the organizer? Four organizer-expressed antagonists have been identified that block canonical Wnt signaling (Figure 1B) $[4-6,12]$. Knockdown of three of the four antagonists reduce head size in Xenopus embryos, which suggests that these antagonists are not simply redundant [6, 12-14]. One interpretation of these results is that Wnt is a very potent signaling molecule and that each antagonist expressed alone at physiological levels is not able to suppress Wnts. Instead, the organizer may need a combination of antagonists to inhibit Wnt signaling sufficiently in order to ensure that the head forms correctly.

The identification of a new Wnt antagonist will now set off a search to find all the places in both embryos and adults that TIKI is expressed, and it will be interesting to see whether these sites are places where other Wnt antagonists are also expressed, indicating that the regulation of Wnt protein levels typically requires multiple regulators acting in concert. It will also be interesting to see whether TIKI is downregulated in cancers known to be Wnt regulated, which would suggest that TIKI can act as a tumor suppressor. Although it is not clear whether TIKI would be a useful therapeutic target as the authors suggest, the results presented here clearly provide an important new addition to our understanding of the many ways Wnt signaling is regulated.

\section{References}

1 Clevers H, Nusse R. Wnt/ $\beta$-catenin signaling and disease. Cell 2012; 149:1192-1205.

2 Angers S, Moon RT. Proximal events in Wnt signal transduction. Nat Rev
Mol Cell Biol 2009; 10:468-477.

3 Niehrs C. Function and biological roles of the Dickkopf family of Wnt modulators. Oncogene 2006; 25:7469-7481.

4 Wang S, Krinks M, Lin K, Luyten FP, Moos M. Frzb, a secreted protein expressed in the Spemann organizer, binds and inhibits Wnt-8. Cell 1997; 88:757-766.

5 Piccolo S, Agius E, Leyns L, et al. The head inducer Cerberus is a multifunctional antagonist of Nodal, BMP and Wnt signals. Nature 1999; 397:707710 .

6 Zhang X, Abreu J, Yokota C, et al. Tikil is required for head formation via Wnt cleavage-oxidation and inactivation. Cell 2012; 149:1565-1577.

7 Niehrs C. Regionally specific induction by the Spemann-Mangold organizer. Nat Rev Genet 2004; 5:425-434.

8 Takada R, Satomi Y, Kurata T, et al. Monounsaturated fatty acid modification of Wnt protein: its role in Wnt secretion. Dev Cell 2006; 11:791-801.

9 Janda CY, Waghray D, Levin AM, Thomas C, Garcia KC. Structural basis of Wnt recognition by Frizzled. Science 2012; 337:59-64.

10 Willert K, Brown JD, Danenberg E, et al. Wnt proteins are lipid-modified and can act as stem cell growth factors. Nature 2003; 423:448-452.

11 Martin BL, Kimelman D. Wnt signaling and the evolution of embryonic posterior development. Curr Biol 2009; 19:R215-R219.

12 Glinka A, Wu W, Delius H, et al. Dickkopf-1 is a member of a new family of secreted proteins and functions in head induction. Nature 1998; 391:357-362.

13 Dickinson AJ, Sive HL. The Wnt antagonists Frzb-1 and Crescent locally regulate basement membrane dissolution in the developing primary mouth. Development 2009; 136:1071-1081.

14 Silva AC, Filipe M, Kuerner KM, Steinbeisser H, Belo JA. Endogenous Cerberus activity is required for anterior head specification in Xenopus. Development 2003; 130:4943-4953. 